http://dx.doi.org/10.1590/1678-4162-8421

Arq. Bras. Med. Vet. Zootec., v.68, n.3, p.673-682, 2016

\title{
Estudo biomecânico comparativo da resistência de segmentos ósseos de rádios de cães autoclavados ou desvitalizados em nitrogênio líquido
}

[Biomechanical comparative study of the strength of bone segments from the radius of dogs autoclaved and devitalized in liquid nitrogen ]

Í.S. Dal-Bó ${ }^{1}$, C.R.A. Ferrigno ${ }^{1}$, C.A.M. Pereira ${ }^{2}$

${ }^{1}$ Faculdade de Medicina Veterinária e Zootecnia - USP - São Paulo, SP

${ }^{2}$ Faculdade de Medicina - USP - São Paulo, SP

\begin{abstract}
RESUMO
O presente estudo comparou, por meio do ensaio de flexão em quatro pontos, a resistência de segmentos de rádios de cães autoclavados e desvitalizados em nitrogênio líquido. Avaliaram-se 40 rádios, que foram divididos em quatro grupos: grupo autoclave (GA), grupo controle autoclave (GCA), grupo nitrogênio (GN) e grupo controle nitrogênio (GCN). Os corpos de prova dos grupos GCA e GCN pertenciam ao lado contralateral dos grupos GA e GN, respectivamente. Os ossos foram autoclavados a $121^{\circ} \mathrm{C}$, por $20 \mathrm{~min}$ a 2atm. A desvitalização em nitrogênio foi realizada por meio da imersão em nitrogênio líquido, por 20min, seguida de descongelamento à temperatura ambiente, sendo finalizada por imersão em solução de NaCL a $0,9 \%$, por mais $15 \mathrm{~min}$. Após os testes biomecânicos, foram comparadas as variáveis força máxima, rigidez, altura máxima à secção transversa do corpo de prova, deslocamento do baricentro em relação ao eixo $\mathrm{x}$, flecha, tensão-tração, tensão-compressão e tensão máxima. Não foram encontradas diferenças estatisticamente significantes entre os grupos GN e GCN. Foram encontradas diferenças estatisticamente significantes entre os grupos GA e GCA somente na comparação da variável força máxima. A análise das variáveis tensão-tração, tensão-compressão e tensão máxima evidenciou diferenças estatisticamente significantes entre os grupos GA e GN, sendo os valores maiores para o grupo GA. Também foi realizada comparação entre os deltas (GN menos GCN e GA menos GCA), em que não foram observadas diferenças estatísticas.
\end{abstract}

Palavras-chave: ensaio de flexão em quarto pontos, osteossarcoma, cirurgia de preservação do membro, ortopedia

\begin{abstract}
The study compared through the four points bending test, the strength of bone segments autoclaved and devitalized in liquid nitrogen radius segments. 40 radius were used, collected and divided into 4 groups, as follows: Autoclave Group (GA), Control Autoclave Group (GCA), Nitrogen Group (NG) and Control Nitrogen Group $(G C N)$. Bone segments from groups GCA and GCN were contralateral to GA and NG. The segments were autoclaved at $121^{\circ} \mathrm{C}$ and 2 atm for 20 minutes. Nitrogen devitalization of the bone segments was obtained by 20 minutes immersion, followed by thawing at room temperature and 15 minutes immersion in $0.9 \% \mathrm{NaCL}$ solution. After biomechanical tests the variables compared were maximum strength, stiffness, maximum hight trough the transverse section of the bone segment, centers of mass dislocation in relation to $x$ axe, arrow, stress-tension, stress-compression and maximum stress. No statistically significant differences between the GN and GCN groups were found. Statistically significant differences were found between GA and GCA groups only in the comparison of the maximum force variable. The analysis of variable stress-tension, stress-compression and maximum stress showed statistically significant differences between the GA and GN groups, with higher values for the GA group. Comparison of the deltas (GN least GCN and GA least GCA) where no statistical differences were observed was also performed.
\end{abstract}

Keywords: four points bending test, osteosarcoma, limb-sparing surgery, orthopaedic

Recebido em 1 de maio de 2015

Aceito em 15 de novembro de 2015

E-mail: idb_vet@yahoo.com.br 


\section{INTRODUÇÃO}

A autoclavagem (Böhm et al., 1998) e a desvitalização em nitrogênio líquido (Rahman et al., 2009) são alguns dos métodos mais utilizados para o tratamento do autoenxerto empregado na cirurgia de preservação do membro ou limb-sparing para tratamento de osteossarcoma em cães. Os princípios dessa cirurgia incluem a ressecção em bloco do tumor e o tratamento dele por meio de técnicas que utilizam o calor ou o frio para desvitalizar as células neoplásicas, e, em seguida, esse segmento é reimplantado e fixado ao osso receptor (Liptak et al., 2006).

Esses métodos de "reciclagem" do osso neoplásico eliminam as células tumorais, entretanto podem alterar suas características biomecânicas. Os processos de autoclavagem e congelamento com nitrogênio líquido são frequentemente utilizados em medicina, principalmente nos países asiáticos, em que a orientação religiosa não permite a utilização de aloenxertos. Ambos apresentam a simplicidade, a praticidade e a disponibilidade imediata dos autoenxertos (Yamamoto et al., 2003; Hayashi et al., 2005; Tsuchiya et al., 2010), contudo o potencial osteoindutor e a matriz orgânica do autoenxerto são perdidos quando o osso é submetido a temperaturas superiores a $60^{\circ} \mathrm{C}$ (Yamamoto et al., 2003; Hayashi et al., 2005). Já o emprego do nitrogênio líquido preserva a resistência biomecânica e mantém as propriedades osteoindutivas e osteocondutivas, além de permitir a reutilização de tecidos moles, como tendões e ligamentos (Sakayama et al., 2006; Rahman et al., 2009; Tanzawa et al., 2009).

O presente trabalho visa comparar biomecanicamente, por meio do ensaio de flexão de quatro pontos, a resistência de segmentos de rádios caninos, tratados com os métodos de autoclavagem e desvitalização em nitrogênio líquido, após seleção das amostras mediante densitometria óptica. A hipótese do trabalho é que os corpos de prova desvitalizados em nitrogênio líquido serão mais resistentes à flexão em quatro pontos do que os autoclavados.

\section{MATERIAL E MÉTODOS}

Este trabalho foi desenvolvido após avaliação da Comissão de Ética no Uso de Animais da FMVZ-USP, sob protocolo ${ }^{\circ} 2795 / 2012$. Foram utilizados 20 cadáveres de cães provenientes do HOVET-FMVZ/USP, cujo óbito não esteve vinculado ao trabalho. Destes, foram coletados 40 rádios.

Como critérios de inclusão, foram estabelecidos cães com ou sem raça definida, com massa corporal acima de $20 \mathrm{~kg}$, machos ou fêmeas, com idade superior a um ano e sem alterações ortopédicas (traumáticas ou neoplásicas) em membros torácicos. Já os critérios de exclusão foram cães que apresentassem afecções ortopédicas macroscópicas (traumáticas ou neoplásicas) envolvendo os membros torácicos ou alterações radiográficas no rádio.

Este estudo foi realizado de forma prospectiva, comparativa e randomizada. Os animais incluídos na pesquisa foram distribuídos aleatoriamente, em grupos, da seguinte forma: para cada cadáver, foi realizado sorteio, que determinou $\mathrm{o}$ tratamento (autoclavagem ou desvitalização em nitrogênio líquido) e qual lado seria submetido a ele. Cada par de rádios pertencia a um tratamento, sendo um lado tratado e o contralateral o seu controle. Obtiveram-se quatro grupos, cada um composto por 10 rádios: grupo autoclave (GA), grupo controle autoclave (GCA), grupo nitrogênio (GN) e grupo controle nitrogênio $(\mathrm{GCN})$.

Os rádios foram coletados em até três horas após o óbito dos animais. Realizou-se desarticulação úmero-rádio-ulnar e carpo-rádio-ulnar. Em seguida, foi feita separação do rádio e da ulna e remoção dos tecidos moles. Foi realizada mensuração do comprimento total do rádio e, logo após, calculou-se a diferença entre esse comprimento e 12 centímetros $(\mathrm{cm})$. $\mathrm{O}$ resultado foi dividido por dois, e o valor obtido em $\mathrm{cm}$ foi o comprimento ósseo removido de cada epífise com a finalidade de preparar o segmento diafisário de $12 \mathrm{~cm}$.

Os corpos de prova do GA foram embalados em papel grau cirúrgico (Cipamed, Brasil) e, durante a autoclavagem, foram submetidos à temperatura de $121^{\circ} \mathrm{C}$ por 20 minutos e à pressão de duas atmosferas (atm) (Sercom, Brasil). 
A desvitalização em nitrogênio líquido consistiu em submergir os segmentos ósseos do GN em nitrogênio líquido por 20 minutos (min). Após, eles passaram pelo processo de descongelamento em temperatura ambiente por $15 \mathrm{~min} \mathrm{e}$, em seguida, foram submersos em solução de $\mathrm{NaCl}$ a $0,9 \%$ também à temperatura ambiente, por mais 15 min.

Todos os corpos de prova foram radiografados com tecnologia computadorizada (Ray Tec, Modelo RT 500/125, Brasil); o regime utilizado foi de $100 \mathrm{~mA}$ e $45 \mathrm{kV}$, foco grosso e tempo de exposição de $2 \mathrm{mAs}$. $O$ exame foi realizado diretamente sobre a mesa, com o corpo de prova posicionado diretamente sobre o cassete. As radiografias foram realizadas nas projeções ML e $\mathrm{CrCa}$. Junto ao cassete e paralelamente ao eixo maior do osso radiografado, foi fixado penetrômetro de alumínio, usado como referência densitométrica (Louzada et al., 1998). A escala de referência era constituída de 20 degraus; o primeiro degrau com 1 milímetro (mm) de espessura, variando a seguir de $1 \mathrm{em}$ $1 \mathrm{~mm}$ até o vigésimo degrau.

A densitometria óptica radiográfica foi realizada da seguinte maneira: as imagens radiográficas foram analisadas em computador, com auxílio do programa ImageJ $1.47 \mathrm{t}^{\circledR}$, a partir do qual foi determinada a densidade óptica óssea em milímetros de alumínio $(\mathrm{mmAl})$. As regiões de interesse para avaliação foram a diafisária média e as metafisárias proximal e distal dos rádios. Esses três pontos foram analisados dentro de um segmento de $12 \mathrm{~cm}$.

As imagens foram salvas em formato JPEG para o processamento no software. Com a ferramenta de seleção de "ponto" do programa, selecionouse um ponto na área central de cada degrau da escala de alumínio e foi aferida a sua tonalidade média de cinzas, que poderia variar de zero (totalmente negro) a 235 (totalmente branco). A sequência de medidas seguiu do primeiro degrau, mais fino (menos radiopaco), para o $20^{\circ}$, mais espesso (mais radiopaco). Esses valores foram armazenados sob a forma de tabelas para cada imagem analisada, a fim de que cada uma tivesse a sua própria escala padrão, evitando a utilização de uma escala única para todas as imagens. Posteriormente, a ferramenta de seleção de área "ponto" foi utilizada para selecionar as regiões diafisária e metafisária de cada imagem radiográfica e medir a sua tonalidade de cinza. Foi realizada a média dos três valores obtidos. Os valores médios de cada exame radiográfico foram registrados nas tabelas, juntamente com as informações de suas respectivas escalas de alumínio, para, então, serem convertidos à unidade de mmAl, mediante a utilização da função "tendência" do software Microsoft Office Excel $2013^{\circledR}$ (Selim, 2013).

A comparação da resistência dos segmentos ósseos dos grupos GA, GCA, GN e GCN foi realizada por meio do ensaio de flexão em quatro pontos, na máquina de ensaios universal (Kratos ${ }^{\circledR}$, modelo KE 3000, Brasil), utilizando-se célula de carga de 3000kgf (quilograma-força) e velocidade de deslocamento de $5 \mathrm{~mm} / \mathrm{min}$. Os parâmetros de segurança definidos na máquina de ensaio foram: carga máxima prevista 3433,5N (350 kgf), deslocamento máximo previsto de $16 \mathrm{~mm}$, pré-carga de $1 \%$ da carga máxima prevista $(34,3 \mathrm{~N})$ e término do ensaio caso a carga de ruptura ultrapasse $70 \%$ da carga máxima prevista. Foi utilizado um dispositivo para ensaio de flexão em quatro pontos. Ele era composto por dois cutelos superiores presos à célula de carga (parte móvel da máquina de ensaios) e por dois cutelos inferiores presos à base da máquina. A distância entre os cutelos era de $25 \mathrm{~mm}$. Cada corpo de prova foi apoiado sobre os cutelos inferiores, com a sua região cranial voltada para cima e com a porção proximal à direitado dispositivo. Para cada par de rádios, primeiramente, foi realizado o ensaio com o corpo de prova sorteado como controle, seguido pelo tratado.

Após os ensaios de flexão em quatro pontos, a porção distal dos rádios fraturados foi preservada. Procedeu-se à ostectomia perpendicular ao eixo longitudinal do osso, e a superfície ostectomizada foi desgastada com o intuito de promover área da secção transversal uniforme. As amostras foram congeladas em freezer a $-20^{\circ} \mathrm{C}$. Em um segundo momento, foram descongeladas à temperatura ambiente, e, sobre a superfície transversal preparada anteriormente, foi aplicada uma camada de primer para metais (Gato Preto, 7 em 1 primer, Brasil). Após 24 horas, foi aplicada uma camada de tinta para tecido (Acrilex, Brasil). Após mais 24 horas, cada osso foi posicionado dentro de um dispositivo de calibração, de forma que a superfície pintada ficasse no mesmo plano do 
dispositivo. Foi utilizada uma câmera fotográfica digital (Câmera digital Canon Powershot G12, 10 megapixels) para fotografar a superfície do osso juntamente com o dispositivo de calibração. O dispositivo de calibração consistia em uma "mesa" com um orifício elíptico em seu centro e quatro marcadores circulares dispostos nos quatro cantos do dispositivo.

Foi desenvolvido um programa de computador na linguagem Delphi 2006 com o intuito de mensurar automaticamente o momento de inércia da superfície óssea pintada; o programa será chamado de PMI (desenvolvido por César Augusto Martins Pereira - Laboratório de Investigação Médica do Sistema Musculoesquelético (LIM-41), Instituto de Ortopedia e Traumatologia, Hospital das Clínicas, Faculdade de Medicina, Universidade de São Paulo, Brasil). Primeiramente, a fotografia digital era carregada e o programa encontrava automaticamente o centro dos quatro marcadores, depois o usuário do programa escolhia manualmente a região colorida de interesse e, após essa etapa, o programa calculava o momento de inércia dos pontos da imagem com a mesma cor escolhida, utilizando as distâncias conhecidas entre cada marcador como referência.

Análise estatística: os valores de idade foram avaliados entre os grupos GA e GN, uma vez que os controles pertenciam ao rádio contralateral do mesmo animal. Foi utilizado o teste $\mathrm{T}$ não pareado, considerando intervalo de confiança $\mathrm{P}<0,05$. Da mesma maneira, os valores de peso foram analisados entre os grupos GA e GN, sendo utilizado o mesmo teste estatístico e o mesmo P. Os valores da densitometria óptica radiográfica foram analisados estatisticamente entre os grupos GN e GCN, GA e GCA, por meio do teste $\mathrm{T}$ pareado, considerando-se $\mathrm{P}<0,05$. A comparação entre os grupos GN e GA foi realizada por meio do teste $\mathrm{T}$ não pareado, considerando-se o mesmo intervalo de confiança.

Os valores obtidos nos testes biomecânicos e momento de inércia foram digitados no programa Excel e posteriormente exportados para o programa SPSS v. 18.0 para análise estatística. Foram descritas as variáveis pela média, mediana, desvio- padrão, mínimo e máximo. Foram comparados os grupos autoclave (GA) e controle (GCA) e nitrogênio (GN) e controle (GCN) pelo teste de Wilcoxon. Para a comparação da variação de cada grupo em relação ao seu controle, calcularam-se os deltas das variáveis (autoclave - controle e nitrogênio controle). Os grupos GA e GN e GCA e GCN foram comparados entre si pelo teste de MannWhitney. Foram ajustados os valores de $\mathrm{P}$ pela técnica de Bonferroni modificada por Finner devido às comparações múltiplas realizadas. Foi considerado um nível de significância de 5\%.

\section{RESULTADOS}

Corpos de prova: foram utilizados 40 rádios provenientes de 20 cadáveres de cães, sendo quatro fêmeas e 16 machos. As médias de idade, peso e medidas dos ossos serão apresentadas nas Tab. 3 e 4. Foram encontradas diferenças estatisticamente significativas durante a comparação dos valores das idades entre os grupos GA e GN, sendo $\mathrm{P}=0,0244$. Já a análise estatística dos valores referentes aos pesos entre os mesmos grupos não revelou diferenças estatisticamente significativas, sendo $\mathrm{P}=0,1851$. Para ambas as análises, foi utilizado teste $\mathrm{T}$ não pareado e $\mathrm{P}<0,05$ (Tab. 1).

Tabela 1. Idade, peso e comprimento total dos grupos, comparando-se GA e GCA e GN e GCN

\begin{tabular}{ccccccc}
\hline & \multicolumn{3}{c}{ GA e GCA } & \multicolumn{3}{c}{ GN e GCN } \\
\hline & $\begin{array}{c}\text { Idade } \\
(\text { anos })\end{array}$ & Peso $(\mathrm{kg})$ & $\begin{array}{c}\text { Comprimento } \\
\text { total do osso } \\
(\mathrm{cm})\end{array}$ & $\begin{array}{c}\text { Idade } \\
(\text { anos })\end{array}$ & Peso (kg) & $\begin{array}{c}\text { Comprimento total } \\
\text { do osso }(\mathrm{cm})\end{array}$ \\
\hline Média & 7,23 & 28,87 & 17,22 & 11,00 & 34,10 & 18,41 \\
DP & 4,47 & 7,22 & 1,80 & 1,89 & 9,59 & 2,37 \\
Mínimo & 1,30 & 20,70 & 15,10 & 8,00 & 22,00 & 12,40 \\
Máximo & 13,00 & 40,00 & 20,90 & 14,00 & 48,00 & 21,00 \\
\hline
\end{tabular}

Densitometria óptica radiográfica: não houve diferença estatística entre os grupos GA e GCA $(\mathrm{P}=0,8119)$ e entre os grupos GN e GCN
$(\mathrm{P}=0,1829)$ analisados pelo teste $\mathrm{T}$ pareado. Igualmente, não foi verificada diferença estatística entre os grupos GA e GN ( $\mathrm{P}=0,7528)$, 
avaliados pelo teste T não pareado. Para os dois testes, foi considerado intervalo de confiança de $5 \%$.

Teste de flexão em quatro pontos e momento de inércia: os dados foram abordados no formato de tabelas, e as variáveis analisadas foram: força máxima (carga aplicada até o limite de escoamento, medida em kgf), rigidez, Hmáx (altura máxima, à secção transversa do corpo de prova, medida em $\mathrm{mm}$ ), Jx (deslocamento do baricentro em relação ao eixo " $\mathrm{x}$ ", medida em $\mathrm{mm}$ elevado à quarta potência $-\mathrm{mm}^{4}$ ), flecha (deslocamento dos atuadores do dispositivo de quatro pontos durante o ensaio de flexão, medida em mm), tensão-tração (tensão gerada na face convexa, medida em megapascal - MPa), tensãocompressão (tensão gerada na face côncava, medida em $\mathrm{MPa}$ ) e tensão máxima (somatório da tensão-tração e da tensão-compressão, igualmente medida em $\mathrm{MPa}$ ).
Foram descritas as variáveis pela média, mediana, desvio-padrão, mínimo e máximo. Foram comparados os grupos autoclave (GA) e controle (GCA) e nitrogênio (GN) e controle (GCN) pelo teste de Wilcoxon. Para a comparação da variação de cada grupo em relação ao seu controle, calcularam-se os deltas das variáveis (autoclave - controle e nitrogênio controle). Os grupos GA e GN e GCA e GCN foram comparados entre si pelo teste de MannWhitney. Ajustaram-se os valores de $\mathrm{P}$ pela técnica de Bonferroni modificada por Finner devido às comparações múltiplas realizadas. Foi considerado um nível de significância de $5 \%$. Não foi encontrada diferença estatisticamente significativa entre os grupos GCA e GCN em nenhum dos parâmetros analisados. A Tab. 2 mostra a comparação entre os grupos GN e GCN. Não foi encontrada diferença estatisticamente significativa entre os grupos.

Tabela 2. Comparação entre os grupos nitrogênio e grupo controle nitrogênio

\begin{tabular}{lcccccccccccc} 
& \multicolumn{4}{c}{ Grupo nitrogênio } & \multicolumn{4}{c}{ Grupo controle nitrogênio } \\
\hline Variáveis & Média & Mediana & Desvio Padrão & Mínimo & Máximo & Média & Mediana & Desvio Padrão & Mínimo & Máximo & P \\
Força máxima (kgf) & 144,48 & 149,10 & 55,99 & 60,60 & 237,60 & 140,76 & 135,00 & 52,24 & 67,80 & 241,50 & 0,816 \\
Rigidez & 45,67 & 43,15 & 23,79 & 17,22 & 92,70 & 50,88 & 47,97 & 21,63 & 29,02 & 102,77 & 0,811 \\
Hmáx (mm) & 6,12 & 6,32 & 1,32 & 4,12 & 8,20 & 6,03 & 6,05 & 1,33 & 4,25 & 8,54 & 0,811 \\
Jx (mm4) & 1182,32 & 1111,37 & 885,54 & 255,41 & 3073,83 & 1114,76 & 1017,12 & 884,80 & 217,35 & 3172,94 & 0,811 \\
Flecha (mm) & 5,21 & 5,19 & 0,47 & 4,29 & 6,07 & 5,20 & 5,01 & 0,79 & 4,40 & 6,66 & 0,957 \\
Momento (N.mm) & 1823,25 & 1863,75 & 676,13 & 757,50 & 2970,00 & 1714,72 & 1601,25 & 710,96 & 847,50 & 3018,75 & 0,811 \\
Tensão-tração (Mpa) & 10,98 & 10,64 & 2,73 & 7,92 & 17,28 & 11,19 & 11,06 & 3,27 & 7,87 & 16,25 & 0,980 \\
Tensão-compressão (Mpa) & 11,32 & 11,25 & 3,22 & 7,47 & 17,97 & 11,63 & 10,23 & 4,13 & 6,86 & 17,27 & 0,980 \\
Tensão máxima (Mpa) & 11,68 & 11,24 & 2,94 & 7,92 & 17,97 & 12,05 & 11,06 & 3,82 & 8,12 & 17,27 & 0,980 \\
\hline
\end{tabular}

Dados comparados pelo teste de Wilcoxon e ajuste de Bonferroni modificado por Finner para os valores de $\mathrm{P}$

Hmáx: altura máxima à secção transversa do corpo de prova

$\mathrm{Jx}$ : deslocamento do baricentro em relação ao eixo "x".

$\mathrm{Na}$ Tab. 3, encontra-se a comparação entre os grupos GA e GCA. Observa-se diferença estatisticamente significativa entre os grupos na variável força máxima, sendo mais altos os valores no grupo autoclave que no grupo controle $(\mathrm{P}=0,044)$.

Na Tab. 4 é apresentada a comparação entre os grupos GN e GA. Houve diferença estatisticamente significativa para as variáveis tensão-tração, tensão-compressão e tensão máxima ( $\mathrm{P}=0,027$ para as três comparações). Os valores das três variáveis foram mais altos no GA.

A Tab. 5 mostra a comparação dos deltas (GN GCN versus GA - GCA). Não houve diferença estatisticamente significativa entre os grupos e os seus controles. Por exemplo, a média de diferença de nitrogênio menos controle na força máxima foi de $3,72 \mathrm{kgf}$ e, no grupo autoclave menos controle, foi de 15,90. Apesar de parecer que a diferença foi maior para o grupo autoclave com o seu controle, essa diferença não foi estatisticamente significativa. Da mesma forma, não houve diferença para o resto das variáveis. 
Tabela 3. Comparação entre os grupos grupo autoclave e grupo controle autoclave

\begin{tabular}{lccccccccccc}
\hline & \multicolumn{4}{c}{ Grupo autoclave } & \multicolumn{6}{c}{ Grupo controle autoclave } \\
\hline Variáveis & Média & Mediana & Desvio Padrão & Mínimo & Máximo & Média & Mediana & Desvio Padrão & Mínimo & Máximo & P \\
Força máxima (kgf) & 141,21 & 151,65 & 43,66 & 70,50 & 222,60 & 125,31 & 132,15 & 45,68 & 66,30 & 213,00 & $\mathbf{0 , 0 4 4}$ \\
Rigidez & 39,36 & 37,07 & 16,43 & 18,59 & 68,67 & 43,39 & 46,65 & 16,38 & 18,09 & 65,47 & 0,238 \\
Hmáx (mm) & 5,13 & 5,05 & 0,87 & 3,94 & 6,92 & 4,97 & 5,02 & 0,76 & 3,80 & 5,93 & 0,422 \\
Jx (mm4) & 570,63 & 523,42 & 307,52 & 183,92 & 1115,60 & 532,93 & 521,21 & 240,37 & 215,28 & 912,12 & 0,445 \\
Flecha (mm) & 6,20 & 5,83 & 1,73 & 3,98 & 9,00 & 5,27 & 4,86 & 1,01 & 4,26 & 7,53 & 0,290 \\
Momento (N.mm) & 1765,13 & 1895,63 & 545,80 & 881,25 & 2782,50 & 1703,75 & 1743,75 & 508,50 & 851,25 & 2662,50 & 0,079 \\
Tensão tração (Mpa) & 16,41 & 15,34 & 5,53 & 9,18 & 30,45 & 14,45 & 14,17 & 3,44 & 9,23 & 22,03 & 0,290 \\
Tensão-compressão (Mpa) & 17,24 & 17,28 & 4,80 & 10,16 & 26,89 & 15,97 & 15,88 & 3,59 & 10,04 & 22,95 & 0,290 \\
Tensão máxima (Mpa) & 17,87 & 17,28 & 5,40 & 10,16 & 30,45 & 16,06 & 15,88 & 3,50 & 10,04 & 22,95 & 0,236
\end{tabular}

Dados comparados pelo teste de Wilcoxon e ajuste de Bonferroni modificado por Finner para os valores de $\mathrm{P}$ Hmáx: altura máxima à secção transversa do corpo de prova Jx: deslocamento do baricentro em relação ao eixo "x".

Tabela 4. Comparação entre os grupos grupo nitrogênio e grupo autoclave

\begin{tabular}{lcccccccccccc} 
& \multicolumn{4}{c}{} & \multicolumn{3}{c}{ Grupo nitrogênio } & \multicolumn{5}{c}{ Grupo controle nitrogênio } \\
\hline Variáveis & Média & Mediana & Desvio Padrão & Mínimo & Máximo & Média & Mediana & Desvio Padrão & Mínimo & Máximo & P \\
Força máxima (kgf) & 144,48 & 149,10 & 55,99 & 60,60 & 237,60 & 141,21 & 151,65 & 43,66 & 70,50 & 222,60 & 0,912 \\
RIGIDEZ & 45,67 & 43,15 & 23,79 & 17,22 & 92,70 & 39,36 & 37,07 & 16,43 & 18,59 & 68,67 & 0,822 \\
Hmáx (mm) & 6,12 & 6,32 & 1,32 & 4,12 & 8,20 & 5,13 & 5,05 & 0,87 & 3,94 & 6,92 & 0,181 \\
Jx (mm4) & 1182,32 & 1111,37 & 885,54 & 255,41 & 3073,83 & 570,63 & 523,42 & 307,52 & 183,92 & 1115,60 & 0,161 \\
Flecha (mm) & 5,21 & 5,19 & 0,47 & 4,29 & 6,07 & 6,20 & 5,83 & 1,73 & 3,98 & 9,00 & 0,347 \\
Momento (N.mm) & 1823,25 & 1863,75 & 676,13 & 757,50 & 2970,00 & 1765,13 & 1895,63 & 545,80 & 881,25 & 2782,50 & 0,884 \\
Tensão-tração (Mpa) & 10,98 & 10,64 & 2,73 & 7,92 & 17,28 & 16,41 & 15,34 & 5,53 & 9,18 & 30,45 & $\mathbf{0 , 0 2 7}$ \\
Tensão-compressão (Mpa) & 11,32 & 11,25 & 3,22 & 7,47 & 17,97 & 17,24 & 17,28 & 4,80 & 10,16 & 26,89 & $\mathbf{0 , 0 2 7}$ \\
Tensão máxima (Mpa) & 11,68 & 11,24 & 2,94 & 7,92 & 17,97 & 17,87 & 17,28 & 5,40 & 10,16 & 30,45 & $\mathbf{0 , 0 2 7}$ \\
\hline
\end{tabular}

Dados comparados pelo teste de Mann-Whitney e ajuste de Bonferroni modificado por Finner para os valores de $\mathrm{P}$ Hmáx: altura máxima à secção transversa do corpo de prova

Jx: deslocamento do baricentro em relação ao eixo "x".

Tabela 5. Comparação dos deltas com respeito ao seu controle entre os grupos grupo nitrogênio e grupo autoclave

\begin{tabular}{lcccccccccccc}
\hline & \multicolumn{4}{c}{ Nitrogênio-controle } & \multicolumn{5}{c}{ Autoclave-controle } \\
\hline Variáveis & Média & Mediana & Desvio Padrão & Mínimo & Máximo & Média & Mediana & Desvio Padrão & Mínimo & Máximo & P \\
Força máxima (kgf) & 3,72 & $-1,35$ & 19,21 & $-21,90$ & 36,00 & 15,90 & 14,85 & 8,60 & 2,40 & 30,90 & 0,732 \\
RIGIDEZ & $-5,21$ & $-5,72$ & 9,60 & $-18,16$ & 9,81 & $-4,03$ & $-6,45$ & 18,92 & $-30,55$ & 43,16 & 0,971 \\
Hmáx (mm) & 0,10 & 0,13 & 0,29 & $-0,34$ & 0,58 & 0,17 & 0,03 & 0,37 & $-0,23$ & 1,00 & 0,833 \\
Jx (mm4) & 67,56 & 23,48 & 132,94 & $-99,11$ & 327,04 & 37,70 & 18,42 & 118,99 & $-78,89$ & 328,66 & 0,773 \\
Flecha (mm) & 0,01 & $-0,11$ & 0,85 & $-1,12$ & 1,67 & 0,93 & 0,04 & 1,75 & $-0,73$ & 4,28 & 0,732 \\
Momento (N.mm) & 65,69 & 15,00 & 238,22 & $-273,75$ & 450,00 & 130,83 & 146,25 & 103,90 & 0,00 & 303,75 & 0,732 \\
Tensão-tração (Mpa) & $-0,20$ & 0,41 & 1,91 & $-3,74$ & 1,83 & 1,82 & 0,97 & 3,10 & $-1,69$ & 8,42 & 0,732 \\
Tensão-compressão (Mpa) & $-0,27$ & 0,48 & 2,00 & $-3,75$ & 1,89 & 1,05 & 1,26 & 2,62 & $-4,49$ & 3,95 & 0,732 \\
Tensão máxima (Mpa) & $-0,28$ & 0,41 & 1,99 & $-3,75$ & 1,83 & 1,66 & 1,31 & 2,93 & $-2,79$ & 7,50 & 0,732 \\
\hline
\end{tabular}

Dados comparados pelo teste de Mann-Whitney e ajuste de Bonferroni modificado por Finner para os valores de $\mathrm{P}$

Hmáx: altura máxima à secção transversa do corpo de prova

$\mathrm{Jx}$ : deslocamento do baricentro em relação ao eixo "x". 


\section{DISCUSSÃO}

A avaliação do status do esqueleto pode ser feita por meio de medidas de densitometria óssea, as quais podem auxiliar na instituição do diagnóstico de osteopenia bem como na monitoração seriada do osso em resposta a afecções, a intervenções cirúrgicas e terapêuticas e em estratégias preventivas relacionadas ao metabolismo ósseo (Muramoto et al., 2005). Por ser um tecido constituído por uma porção mineral e outra orgânica, o osso pode ser avaliado não só por métodos de imagem, que quantificam indiretamente esse conteúdo mineral, como também por métodos que envolvam ensaios mecânicos (Louzada et al., 2006). No presente trabalho, optou-se pela densitometria óptica radiográfica como método de homogeneizar e diminuir a possibilidade de manter corpos de prova osteopênicos na amostra. Sendo assim, a análise biomecânica foi realizada preocupando-se apenas com a resistência ao ensaio de flexão em quatro pontos e com os efeitos dos tratamentos a que os corpos de prova foram submetidos, uma vez que a densitometria óptica já havia sido analisada previamente.

A densitometria óptica radiográfica se mostrou ferramenta simples, prática, com valor acessível, e foi utilizada como método de comparação entre os grupos GN e GCN, GA e GCA e entre GN e GA. Muramoto et al. (2005) mencionaram que a variável peso pode influenciar a densidade óssea, apresentando correlação positiva, quando analisaram e estabeleceram valores de densidade em rádios de cães da raça Poodle, por meio da densitometria óptica radiográfica. Os mesmos autores encontraram fraca correlação entre a idade e a densidade óptica. No presente estudo, os valores absolutos, referentes ao peso, foram maiores nos grupos GN e GCN, porém não houve diferença significativa entre os grupos GA e GN. A média de idade também foi maior no grupo nitrogênio, havendo diferença estatisticamente significativa entre os mesmos grupos. Não obstante esses achados, não foram encontradas diferenças estatisticamente significativas entre os valores de densitometria, durante a análise dos grupos GN e GCN, GA e GCA e GN e GA, o que discorda de Muramoto et al. (2005) e mostra que houve homogeneidade entre as amostras com relação à densitometria óptica.
Os grupos GN e GCN (mesmo animal) apresentaram maiores médias de peso e idade, apesar de o estudo ser realizado de forma aleatória, em que o sorteio do tratamento e do lado em que a técnica iria ser executada ser feito antes da coleta das amostras. Não houve, todavia, diferenças estatisticamente significativas quanto a essas variáveis.

Pesquisas semelhantes a este trabalho realizaram comparação da resistência óssea por meio de testes biomecânicos de compressão e torção (Köhler et al., 1986; Duarte e Schaeffer, 2000; Yamamoto et al., 2003). No presente estudo, optou-se pela utilização do teste de flexão em quatro pontos, por se tratar de um osso longo e para tentar mimetizar a aplicação de forças na superfície cranial dos rádios, como na cirurgia de limb-sparing, em que o implante, em geral uma placa, é aplicado nessa região (Liptak et al., 2006). Outra razão para que fosse escolhido o ensaio de flexão é que as placas são menos resistentes às forças de arqueamento e essa força é predominante em relação às de torção ou compressão no rádio canino. Além disso, o teste de quatro pontos distribui a força de maneira uniforme entre os pontos de contato. Os pontos de falha do corpo de prova tendem a residir ao longo dessa região, porém longe dos pontos de contato, permitindo avaliação mais fidedigna (Draper e Goodship, 2003).

Em última análise, foram feitos os cálculos do momento de inércia nos corpos de prova após o ensaio destrutivo de flexão. Esses valores permitem compreender e mesmo transferir parcialmente os dados do ensaio, como a resistência real do corpo em qualquer força aplicada na forma de arqueamento do rádio do cão, permitindo supor que, em diferentes regiões de aplicação de força no rádio, a resistência final do osso será muito semelhante à encontrada no estudo. Portanto, a pesquisa ligada não somente à resistência da força máxima mas também ao estudo da área e da geometria do corpo de prova se mostrou importante a este projeto, pois os osteossarcomas podem afetar diferentes áreas do rádio do cão, principalmente sua região distal.

A utilização do programa computacional PMI, desenvolvido especialmente para essa pesquisa, foi fundamental para facilitar o cálculo do momento de inércia, uma vez que a amostra utilizada, por motivos éticos, não era composta 
de cães experimentais. Portanto, havia rádios de diferentes conformações, dependendo do porte e da raça dos animais, o que dificultou a padronização do tipo de cálculo dessa variável, visto que nem todos os rádios, à secção transversa, apresentavam formato elíptico. Barbosa et al. (2010) e Reis et al. (2011), contudo, usaram o método do cálculo do momento de inércia para uma elipse oca, visando à avaliação biomecânica de fêmures e tibiotarsos de frango, respectivamente. Tal método foi possível, pois esses autores utilizaram uma amostra homogênea, composta por frangos de corte, do mesmo lote, com peso e idade semelhantes, portanto, com conformação óssea semelhante, o que facilitou o cálculo dessa variável.

Singh et al. (2010) realizaram análise histopatológica e biomecânica, por meio do teste de compressão, de segmentos ósseos humanos submetidos aos métodos de esterilização por autoclavagem, fervura, pasteurização e irradiação, com a finalidade de determinar qual deles era capaz de destruir as células tumorais sem comprometer as propriedades biomecânicas. Eles observaram que, apesar de todos os métodos terem sido eficazes em relação à eliminação das células neoplásicas, as propriedades mecânicas foram prejudicadas e a diminuição da resistência dos corpos de prova foi diretamente proporcional ao aumento da temperatura.

A hipótese do presente trabalho era de que o processo de autoclavagem diminuiria a resistência dos segmentos de rádio caninos, porém isso não foi comprovado, sendo essa técnica adequada para preservação dos segmentos ósseos quanto ao aspecto biomecânico, como afirmaram Köhler et al. (1986). Entretanto, de acordo com Singh et al. (2010), o calor modifica as propriedades do colágeno presente no tecido ósseo, o que faz com que essa proteína sofra desnaturação à temperatura de $100^{\circ} \mathrm{C}$ e ocorra diminuição da resistência biomecânica. A estrutura do colágeno, contudo, permanece inalterada até a temperatura de $60^{\circ} \mathrm{C}$.

Köhler et al. (1986) avaliaram as propriedades biomecânicas, de ossos longos de coelhos autoclavados, por meio do teste de torção. Eles utilizaram os seguintes protocolos de autoclavagem: $110^{\circ} \mathrm{C}$ por $225 \mathrm{~min}, 121^{\circ} \mathrm{C}$ por
$20 \mathrm{~min}$ e $131^{\circ} \mathrm{C}$ por $2 \mathrm{~min}$ e observaram diminuição acentuada da resistência e da rigidez nos ossos autoclavados a baixas temperaturas e por tempo prolongado. Além disso, foi observada diminuição do peso dos ossos, possivelmente devido à perda de água e à desnaturação do colágeno. A escolha do protocolo de autoclavagem foi baseada nos achados de Köhler et al. (1986) e nos regimes disponíveis na autoclave do Serviço de Cirurgia de Pequenos Animais do HOVET-FMVZ/USP.

Dohtsu et al. (2001) compararam ossos a fresco aos que passaram pelo processo de pasteurização, fervura ou congelamento em nitrogênio líquido, em ratos. O congelamento foi realizado em três imersões em nitrogênio líquido de três minutos, com intervalo de cinco minutos à temperatura ambiente. Corroborando os autores, igualmente não foram encontradas diferenças estatisticamente significativas entre os grupos GN e GCN quanto à resistência biomecânica, demonstrando que a desvitalização em nitrogênio líquido não compromete essa propriedade.

Voggenreiter et al. (1994) submeteram tíbias de ratos aos tratamentos de congelamento a $-70^{\circ} \mathrm{C}$, radiação gama, autoclavagem a $134^{\circ} \mathrm{C}$ por $3 \mathrm{e}$ 5 min e liofilização. Eles observaram os corpos de prova ao microscópio eletrônico e encontraram alterações na microestrutura das amostras autoclavadas e liofilizadas. As últimas apresentaram microfraturas e a primeira, engrossamento das fibras da matriz quando a autoclavagem durou $3 \mathrm{~min}$, bem como desnaturação da matéria orgânica aos 5 min de autoclavagem. Essa pode ser a razão da redução da estabilidade biomecânica e da perda da capacidade osteocondutora. Não foi realizada análise microscópica no presente estudo, contudo o aspecto macroscópico dos corpos de prova autoclavados evidenciava alteração em sua estrutura, ou seja, eles apresentavam aspecto "ressecado". Da mesma maneira, apesar de não haver diferença estatística entre os deltas, o comportamento "visual" do corpo de prova ao ensaio de flexão denotava perda da flexibilidade/maior fragilidade em comparação ao grupo nitrogênio. Os segmentos ósseos do grupo GN sofriam acentuada curvatura, em sentido craniocaudal, antes da falha, enquanto os do grupo GA não sofriam esse tipo de deformação e respondiam à aplicação da carga 
"explodindo" e sofrendo fraturas cominutivas. Apesar desses resultados, foram observadas, durante a comparação entre os grupos GN e GA, diferenças estatisticamente significativas, em relação às variáveis tensão-tração, tensãocompressão e tensão máxima, sendo os valores superiores para o GA, o que contraria a hipótese do estudo. Não foi possível estabelecer uma justificativa para tais resultados, sugerindo-se novos estudos com número amostral superior.

\section{CONCLUSÃO}

A comparação biomecânica, por meio do ensaio de flexão em quatro pontos, de segmentos de rádios caninos submetidos à autoclavagem e à desvitalização em nitrogênio líquido, não demonstrou diferenças estatisticamente significativas. Portanto, concluiu-se que ambos os métodos podem ser utilizados para $\mathrm{o}$ tratamento de autoenxertos para a cirurgia de preservação do membro, sem prejuízo quanto à resistência biomecânica.

\section{REFERÊNCIAS}

BARBOSA, A.A.; MORAES, G.H.K.; TORRES, R.A. et al. Avaliação da qualidade óssea mediante parâmetros morfométricos, bioquímicos e biomecânicos em frangos de corte. Rev. Bras. Zootec., v.39, p.772-778, 2010

BÖHM, P.; SPRINGFELD R.; SPRINGER H. Re-implantation of autoclaved bone segments in musculoskeletal tumor surgery. Clinical experience in 9 patients followed for 1.1-8.4 years and review of the literature. Arch. Orthop. Trauma Surg., v.118, p.57-65, 1998.

DOHTSU, T.; INOKUCHI, T.; HIRONAKA, R. et al. Experimental study on healing of bone implants treated by heating or freezing. J. Oral Pathol. Med., v.6, p.43-50, 2001.

DRAPER, E.C.; GOODSHIP, A.E. A novel technique for four-point bending of small bone samples with semi-automatic analysis. $J$. Biomech., v.36, p.1497-1502, 2003.

DUARTE, L.S.; SCHAEFFER, L. Comparação da resistência à compressão de ossos bovinos congelados e liofilizados. Rev. Bras. Eng. Bioméd., v.16, p.89-93, 2000.
HAYASHI, K.; TSUCHIYA, H.; YAMAMOTO, N. et al. Histological examination of autoclaved bone removed 12 years after it was transplanted. J. Orthop. Sci., v.10, p.425-429, 2005.

KÖHLER, P.; KREICBERGS, A.; STRÖMBERG, L. Physical properties of autoclaved bone. Torsion test of rabbit diaphyseal bone. Acta Orthop. Scand., v.57, p.141-145, 1986.

LIPTAK, J.M.; DERNELL, W.S.; EHRHART, N. et al. Cortical allograft and endoprosthesis for limb-sparing surgery in dogs with distal radial osteosarcoma: a prospective clinical comparison of two different limb-sparing techniques. Vet. Surg., v.35, p.518-533, 2006.

LOUZADA, M.J.Q.; PELÁ, C.A.; BELANGERO, W.D. et al. Metodologia para avaliação de densidade em imagem radiográfica. RBE-Cad. Eng. Bioméd., v.14, p.37-47, 1998.

LOUZADA, M.J.Q.; BELANGERO, W.D.; MARTINS, L.E. B. Avaliação de parâmetros biomecânicos pela densitometria radiográfica. Estudo "in vitro" em osso cortical de tíbias de carneiros. Rev. Bras. Biomec., v.7, p.33-38, 2006.

MURAMOTO, C.; STERMAN, F.A.; PINTO, A.C.B.F. Estabelecimento de valores de densidade mineral óssea (DMO) das regiões metafisária e diafisária do rádio de cães da raça Poodle por meio da densitometria óptica radiográfica. Braz. J. Vet. Res. Anim. Sci., v.42, p.89-97, 2005.

RAHMAN, M.A.; BASSIONI, A.; SHALABY, H. Reimplantation of the resected tumourbearing segment after recycling using liquid nitrogen for osteossarcoma. Int. Orthop., v.33, p.1365-1370, 2009.

REIS, D.T.C.; TORRES, R.A.; BARBOSA, A.A. et al. Efeito de linhagem e sexo nas características geométricas e biomecânicas de tíbias de frangos de corte. Acta. Sci. Anim. Sci., v.33, p.101-108, 2011.

SAKAYAMA, K.; TSUCHIYA, H.; FUJIBUCHI, T. et al. Pathological findings of an autograft containing osteosarcoma treated by liquid nitrogen retrieved 2 years after implantation. $J$. Orthop. Sci., v.11, p.655-656, 2006. 
SELIM, M.B. Avaliação estrutural do osso terceiro metacarpiano equino frente à implantação do biopolímero à base de mamona. 2013. 124f. Dissertação (Mestrado em Ciências) - Faculdade de Medicina Veterinária e Zootecnia, Universidade de São Paulo, São Paulo, SP.

SINGH, V.A.; NAGALINGAM, J.; SAAD, M. et al. Which is the best method of sterilization of tumor bone for reimplantation? A biomechanical and histopathological study. BioMed. Eng. OnLine, v.9, p.1-15, 2010.

TANZAWA, Y.; TSUCHIYA, H.; SHIRAI, T. et al. Histological examination of frozen autograft treated by liquid nitrogen removed after implantation. J. Orthop. Sci., v.14, p.761-768, 2009.
TSUCHIYA, H.; NISHIDA, H.; SRISAWAT, P. et al. Pedicle frozen autograft reconstruction in malignant bone tumors. J. Orthop. Sci., v.15, p.340-349, 2010.

VOGGENREITER, G.; ASCHERL, R.; BLÜMEL, G. et al. Effects of preservation and sterilization on cortical bones grafts. A scanning electron microscopic study. Arch. Orthop. Trauma Surg., v.113, p.294-296, 1994.

YAMAMOTO, N.; TSUCHIYA, H.; NOJIMA, T. et al. Histological and radiological analysis of autoclaved bone 2 years after extirpation. $J$. Orthop. Sci., v.8, p.16-19, 2003. 